\title{
Polidactilismo unilateral incomum em equino: relato de caso
}

\author{
[Unusual unilateral polydactylism in a horse: a case report] \\ A.A.T. Nascimento ${ }^{1}$, G.D. Carvalho ${ }^{2 *}$, C.M. Sássi $i^{3}$, A. Lang $^{1}$ \\ ${ }^{1}$ Faculdade de Ciências Biológicas e da Saúde - FACISA/UNIVIÇOSA - Viçosa, MG \\ ${ }^{2}$ Instituto Federal Norte de Minas Gerais - IFNMG Campus Salinas - Salinas, MG \\ ${ }^{3}$ Instituto Ecológico Búzios Mata Atlântica - IEBMA - Búzios, RJ
}

\begin{abstract}
RESUMO
A polidactilia é um defeito genético caracterizado pela duplicação parcial ou completa de um dígito. Estudos em humanos, bovinos, cães e gatos indicam que um gene autossômico dominante de penetração incompleta é o responsável por essa alteração. A polidactilia é rara em equinos e, nessa espécie, sua causa ainda não foi esclarecida. Entretanto, quando ocorre, está relacionada à má formação congênita da falange. Este trabalho descreve, por meio da observação de exames físico, radiográfico e ultrassonográfico, a ocorrência de polidactilia unilateral, com características incomuns, em um equino de nove anos de idade. $\mathrm{O}$ animal, com um histórico de claudicação intensa, apresentava, desde o nascimento, divisão completa do dígito do membro torácico direito, com simetria entre as duas porções distais. Ao exame radiográfico, identificou-se a presença de dois dígitos separados e de dimensões semelhantes, sendo esta uma manifestação incomum de polidactilia na espécie equina.
\end{abstract}

Palavras-chave: deformidade congênita, equino, polidactilia

\begin{abstract}
Polydactyly is a genetic disorder characterized by partial or complete duplication of a digit. Studies in humans, cattle, dogs and cats indicate that an autosomal dominant gene of incomplete penetrance is responsible for this change. Polydactyly is rare in horses and its cause remains unclear. However, when it occurs it is related to congenital malformation of the phalanx. This paper describes, through the observation of physical, radiographic and ultrasound finds, the occurrence of unilateral polydactyly, with unusual features in a nine year old horse. Since birth, the animal, which had a history of severe lameness, had complete division of the digit of the right forelimb, with symmetry between the two distal portions. The radiographic examination identified the presence of two separated digits with similar size, which is an unusual manifestation of polydactyly in the equine species.
\end{abstract}

Keywords: congenital anomaly, equine, polydactyly

\section{INTRODUÇÃO}

A polidactilia é um defeito genético caracterizado pela duplicação parcial ou completa de um dígito (Welch, 1999; Carstanjen et al., 2007).

Esta anomalia é descrita no homem e em alguns animais, como gatos (Sis e Getty, 1968), cães (Hansen, 1972; Jubb et al., 1985; Villagomez e

Recebido em 23 de maio de 2011

Aceito em 9 de maio de 2012

*Autor para correspondência (corresponding author)

E-mail: gabriel.carvalho@ifnmg.edu.br
Alonso, 1998), bovinos (Herschler et al., 1962; Leipold et al., 1972; Guffy e Leipold, 1977; Johnson et al., 1981; Johnson et al., 1982; Mather, 1987; Vermunt et al., 2000; Murondoti e Busayi, 2001; Bahr et al., 2003; Alam et al., 2007; Carstanjen et al., 2010), caprinos (Al-Ani et al., 1997; Gutierrez et al., 2000), ovinos (Dennis e Leipold, 1979), camelídeos (Fowler, 1989; Johnson e Gentz, 1990; Bani-Ismail et al., 1999; Strauss, 2002; Altenbrunner-Martinek et al., 2007; Zapata et al., 2008), suínos 
(Whittington et al., 1986; Huston, 1993) e equinos (McGavin e Leipold, 1975; Behrens et al., 1979; Silveira e Lazzeri, 1985; Crowe e Swerczek, 1985; Stanek e Hantak, 1986; Barber, 1990; Colboume et al., 1991; Weinhart et al., 1996; Welch, 1999; Giofré et al., 2004; Carstanjen et al., 2007).

Também conhecida como hiperdactilia, corresponde à existência de dedos supranumerários e pertence a um grupo heterogêneo de anomalias que ocorre em muitos animais, principalmente mamíferos, como resultado de mutações espontâneas, de transmissão genética (herdáveis ao longo das gerações). Há pelo menos 92 genótipos diferentes relacionados ao fenótipo da polidactilia em animais, e o tipo de herança pode ser recessivo, dominante ou codominante (Mustacchi, 2000).

Em humanos, essa anomalia é classificada como pré-axial, central ou pós-axial. A polidactilia préaxial ocorre uma vez a cada 12.000 nascidos vivos, a polidactilia central é a forma menos comum e a pós-axial é a forma de alteração mais comum, ocorrendo cerca de oito vezes mais que a primeira (Weinstein, 2000). Nos gatos é um traço autossômico dominante (Jezyk, 1985). Em equinos, sua causa ainda não foi esclarecida (Carstanjen et al., 2007). Estudos de afecções em humanos, bovinos, cães e gatos indicam um gene autossômico dominante de penetração incompleta (Behrens et al., 1979). De acordo com Stanek e Hantak (1986), a polidactilia é rara em equinos, entretanto esta anomalia representa a má formação congênita de falange mais frequente nessa espécie. Em equinos há relatos de casos de polidactilismo bilateral em região medial de membro (Stanek e Hantak, 1986; Carstanjen et al., 2007).

O presente trabalho relata o atendimento de um equino macho, de nove anos de idade, com histórico de claudicação intensa e divisão completa do dígito do membro torácico direito, com simetria entre as duas porções distais, configurando um quadro incomum de polidactilia unilateral na espécie equina.

\section{CASUÍSTICA}

Um equino macho inteiro, da raça Campolina, com nove anos de idade, foi atendido no Setor de Clínica Médica e Cirurgia de Grandes Animais do Hospital Veterinário da Univiçosa com um histórico de claudicação e alteração física congênita do membro torácico direito (MTD) (Fig. 1).

No exame físico, observou-se escore corporal 2 (escala de 0 a 5), comportamento e parâmetros vitais normais. A alteração física consistiu na presença de dois dígitos completos, de dimensões semelhantes, a partir da articulação metacarpofalangeana do MTD (Fig. 2), com hiperextensão das articulações interfalangeanas proximal e distal. $\mathrm{O}$ animal apresentava ainda deformidade dos cascos, com extensão pronunciada das pinças, limitação de movimentos distais à articulação metacarpofalangeana, hipotrofia muscular em todo o membro e claudicação grau III (escala de I a IV).

Ao exame radiográfico, nas projeções dorsopalmar e lateromedial, identificou-se a presença de apenas um osso III metacarpiano e dois sesamoides proximais, como em animais normais, mas as falanges proximal, intermédia e distal, além dos sesamoides distais, apresentavam-se em duplicata, formando dois dígitos separados; a mesma alteração foi observada na projeção posteroanterior. As falanges evidenciavam deformidade e reações de osteólise e proliferação óssea, principalmente na segunda falange, e sinais de fusão nas articulações interfalangeanas proximal e distal (Fig. 3)

O exame ultrassonográfico evidenciou a presença dos tendões flexores superficial e profundo, ligamento acessório do tendão flexor digital profundo e ligamento músculo-interósseo com características normais. Alterações ultrassonográficas evidentes consistiram em áreas de maior ecogenicidade, compatível com excesso de tecido conjuntivo fibroso e áreas hipo ou anecoicas compatíveis com acúmulo de líquido e vasodilatação. 
Polidactilismo unilateral...

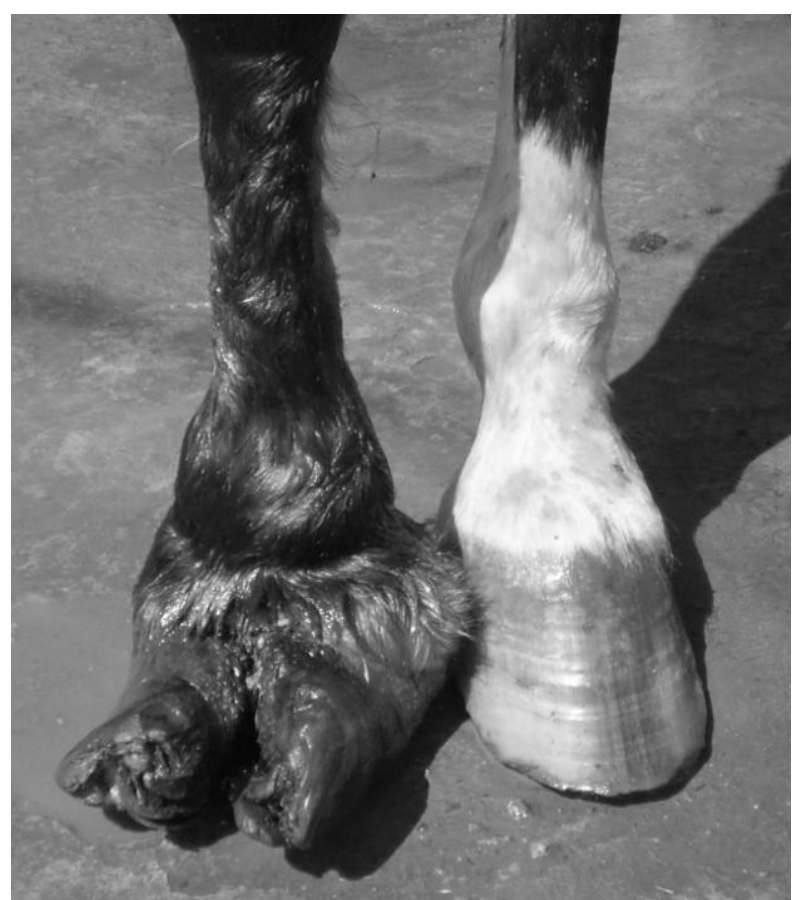

Figura 1. Vista frontal da alteração física congênita do membro torácico direito, com presença de dois dígitos.

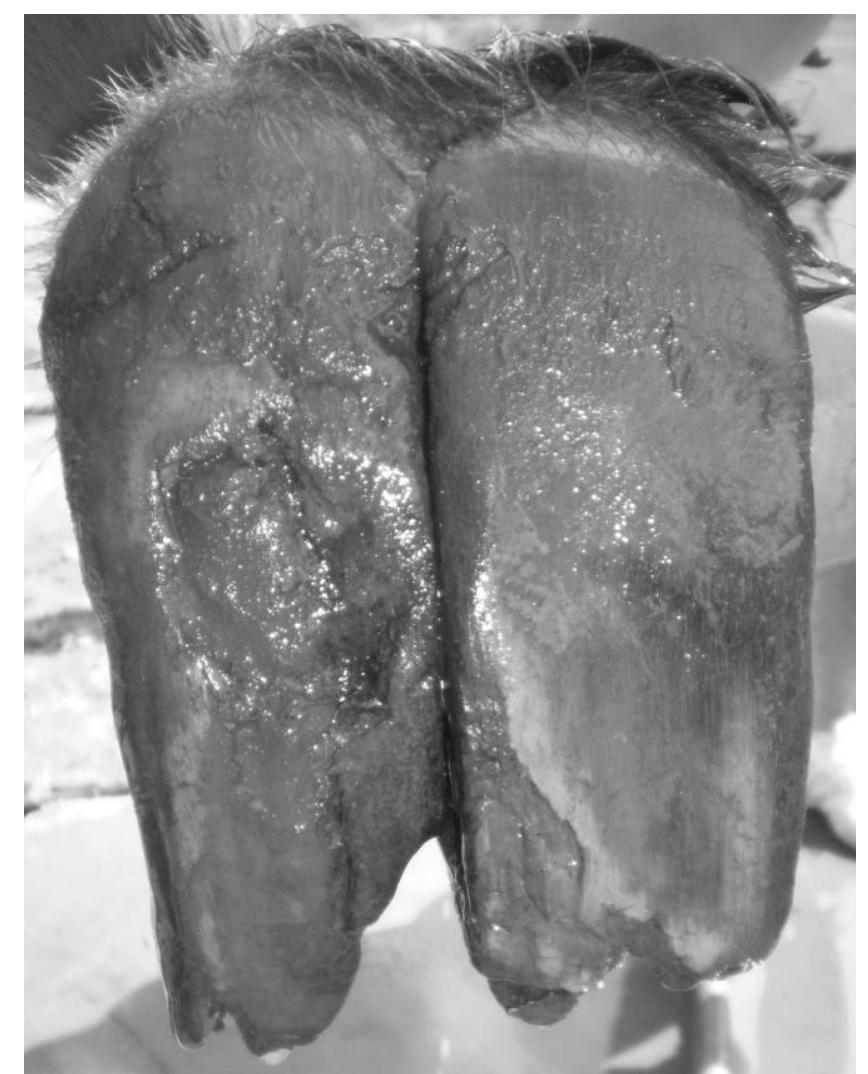

Figura 2. Casco visto pela face plantar. Presença de dois dígitos separados, de dimensões semelhantes. 


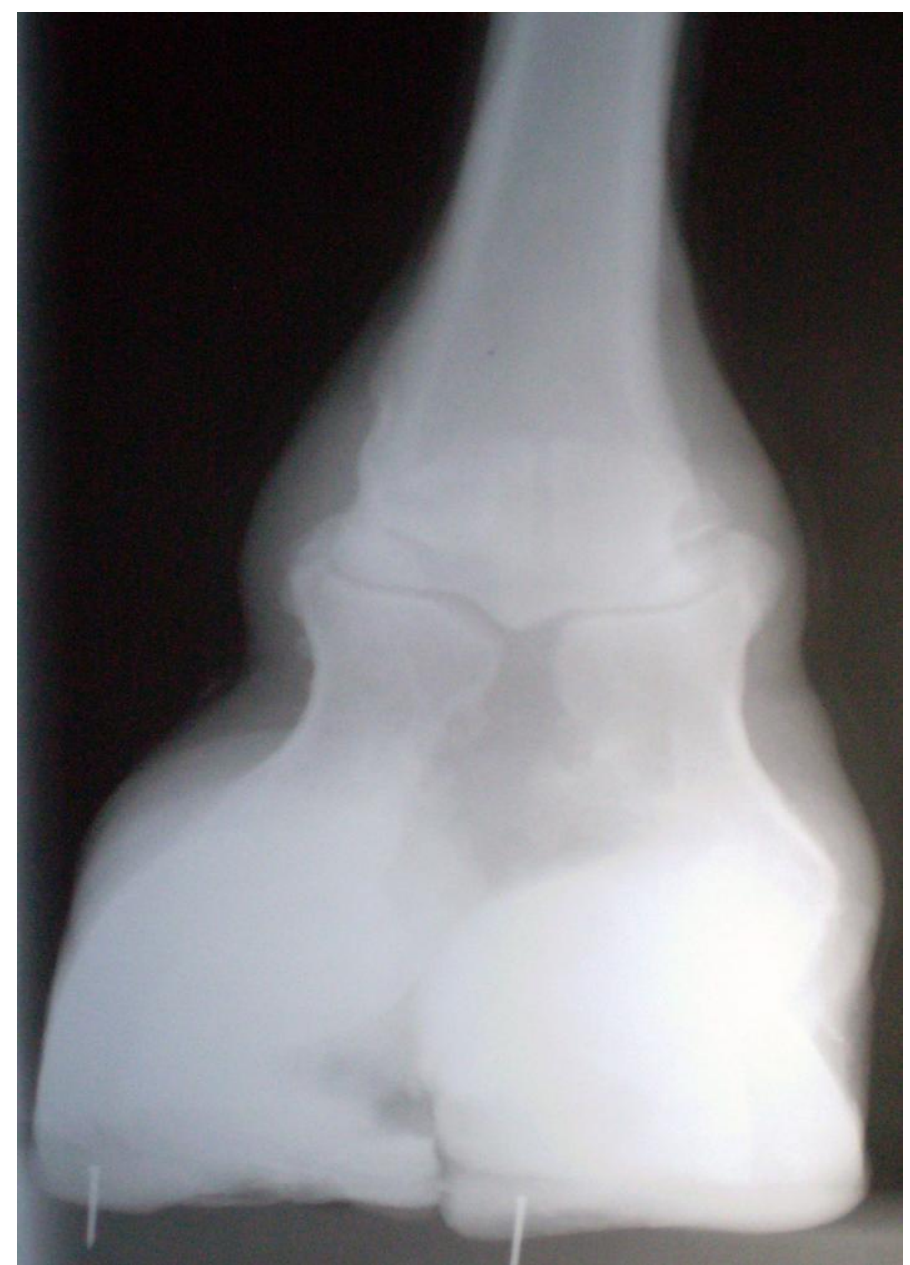

Figura 3. Imagem radiográfica apresentando as estruturas ósseas em duplicata. Projeção posteroanterior.

\section{DISCUSSÃO}

O diagnóstico de polidactilia é normalmente baseado no exame clínico. Entretanto, uma avaliação radiográfica do metacarpo e das regiões adjacentes é importante para determinar as anormalidades (Barber, 1990) ósseas e a presença de MC I supranuméricos (Behrens et al., 1979). Os achados dos exames físico, radiográfico e ultrassonográfico relatados neste caso são compatíveis com uma manifestação incomum de polidactilismo, pela presença de dois dígitos completos e de dimensões semelhantes, na porção distal do membro.

Em equinos, o dígito supranumérico é observado nos membros torácicos, em aproximadamente $80 \%$ dos casos, principalmente na região medial do membro (Evans et al. (1965) apud Stanek e Hantak, 1986), apesar de a ocorrência de dois dígitos supranuméricos em uma extremidade de membro já ter sido relatada por Wilson (1927) apud Stanek e Hantak (1986).

O tratamento indicado para casos de polidactilia é a retirada do dígito extra, com preservação das estruturas do dígito principal (Welch, 1999). Essa recomendação é usual em casos de dígitos supranumérios mediais (Stanek e Hantak, 1986; Carstanjen et al., 2007). Porém, no caso apresentado, os dígitos apresentam dimensões semelhantes e são essenciais para a movimentação do animal, devido a sua localização distal. McGavin e Leipold (1975) consideram que a remoção cirúrgica dos dígitos supranumerários é por questão estética e para prevenir injúrias no dígito.

Apesar de a polidactilia ser um defeito genético (Welch, 1999; Mustacchi, 2000), segundo relatos 
do proprietário, o animal produziu descendentes sem qualquer alteração física, sendo ainda utilizado como reprodutor. Há autores que sugerem a possibilidade da transmissão hereditária da polidactilia, mas não há evidências de literatura que confirmem tal fato (Stanek e Hantak, 1986).

Estudos aprofundados sobre tal deformidade congênita e sua relação genética se fazem necessários para sua compreensão na espécie equina.

\section{REFERÊNCIAS}

AL-ANI, F.K.; HAILAT, N.Q.; FATHALLA, M.A. Polydactyly in Shami breed goats in Jordan. Small Rum. Res., v.26, p.177-179, 1997.

ALAM, M.R.; LEE, J.I.; LEE, H.B. et al. Supernumerary ectopic limbs in Korean indigenous cattle: four case reports. Vet. Med., v.52, p.202-206, 2007.

ALTENBRUNNER-MARTINEK， B.; KLEIN， D.; KOFLER, J.; BAUMGARTNER, W. Kongenitale Extremitätenmissbildungen bei einem Lama (Lama glama): Polydactylie kombiniert mit Arthrogryposis. Berl. Münch. Tierärztl. Wschr., v.120, p.508-512, 2007.

BANI-ISMAIL, Z.; HAWKINS, J.F.; SIEMS, J.J. Surgical correction of polydactyly in a camel (Camelus dromedarius). J. Zoo Wildife Med., v.30, p.301-304, 1999.

BAHR, C.; WITTENBERG, K.; DISTL, O. Case report: polydactyly in a German holstein calf. Dtsch. Tierärztl. Wochenschr, v.110, p.330-335, 2003.

BARBER, S.M. Unusual polydactylism in a foal: a case report. Vet. Surg., v.19, p.203-207, 1990.

BEHRENS, E.; DONAWICK, W.J.; RAKER, C.W.; WOODHEAD, J.S. Polydactylism in a foal. J. Am. Vet. Med. Assoc., v.174, p.266-268, 1979.

CARSTANJEN, B.; ABITBO, M.; DESBOIS, C. Bilateral polydactyly in a foal. $J$. Vet. Sci., v.8, p.201203, 2007.

CARSTANJEN, B.; PENNECKE, J.; BOEHART, S.; MÜLLER, K.E. Unilateral Polydactylism in a German Holstein-Friesian Calf - A case report. Thai J. Vet. Med. v.40, p.69-74, 2010.

COLBOUME, C.M.; YOVICH, J.V.; TURNER, AS.; EASLY, K.J. Surgical treatment of polydactylism and long term follow up in five horses. Aust. Equine Vet. J., v.14, p.43-146, 1991.
CROWE, M.W.; SWERCZEK, T.W. Equine congenital defects. Am. J. Vet. Res., v.46, p.353-358, 1985.

DENNIS, S.M.; LEIPOLD, H.W. Ovine congenital defects. Vet. Bull., v.49, p.233-239, 1979.

FOWLER, M. Congenital Hereditary Conditions. Medicine and Surgery of South American Camelids. Iowa: Iowa State University Press, 1989, p.337-365.

GIOFRÉ, F.; CARACCIOLO, V.; ZANOTTI, M. et al. Polydactyly in a Murgese horse: a case report. $J$. Equine Vet. Sci., v.24, p.248-250, 2004.

GUFFY, M.; LEIPOLD, H.W. Radiology diagnosis of economically important genetic defects in cattle. $J$. Am. Radiol. Sot., v.18, p.109-116, 1977.

GUTIERREZ, C.; RODRIGUEZ, J.L.; CASTELLANO, E. et al. Multiple malformations in a newborn goat. Can. Vet. J., v.41, p.568-569, 2000.

HANSEN, J.S. Lateral polydactylism in the dog. Vet. Med. Small Anim. Clin., v.67, p.536, 1972.

HERSCHLER, M.S.; FECHHEIMER, N.S.; GILMORE, L.O. Congenital abnormalities in cattle: their association with hereditary and environmental factors. J. Dairy Sci., v.45, p.1493-1499, 1962.

HUSTON, K. Heritability and diagnosis of congenital abnormalities in food animals. Vet. Clin. North Am.: Food Anim. Pratt., v.9, p.1-9, 1993.

JEZYK, P.F. Constitutional Disorders of the skeleton in dogs and cats. In: NEWTON, C.D.; NUNAMAKER, D,M. Textbook of Small Animal Othopaedics. Philadelphia: JB lippincott, 1985. p.637654.

JOHNSON, J.L.; LEIPOLD, H.W.; SCHALLES, R.R. et al. Hereditary polydactyly in Simmental cattle. $J$. Hered, v.72, p.205-208, 1981.

JOHNSON, J.L.; LEIPOLD, J.W.; GUFFY, M.M. et al. Characterization of bovine polydactyly. Bovine Pract., v.3, p.7-14, 1982.

JOHNSON, L.W.; GENTZ, E.J. Multiple nonlethal congenital anomalies in a llama. J. Am. Vet. Med. Assoc., v.96, p.630-631, 1990.

JUBB, K.V.F.; KENNEDY, P.C.; PALMER, N. Pathology of Domestic Animals. 3.ed. v.I. New York: Academic Press, 1985. p.28.

LEIPOLD, H.W.; DENNIS, S.M.; HUSTON, K. Polydactyly in cattle. Cornell Vet., v.62, p.337-345, 1972.

MATHER, D.B. Polydactyly in calves. Vet. Rec., v.120, p.487, 1987. 
McGAVIN, M.D.; LEIPOLD, H.W. Attempted surgical correction of equine polydactylism. $J$. Am. Vet. Med. Assoc., v.166, p.63-64, 1975.

MURONDOTI, A.; BUSAYI, R.M. Perineomelia, polydactyly and other malformations in a Mashona calf. Vet. Rec., v.148, p.512-513, 2001.

MUSTACCHI, Z; PERES, S. Genética Baseada em Evidências: Síndromes e heranças. São Paulo: CID Editora Ltda, 2000. 1299p.

SILVEIRA, J.M.; LAZZERI, L. Sobre um caso de polidactilia em equino. Arq. Bras. Med. Vet. Zootec., v.37, p.241-247, 1985.

SIS, R.F.; GETTY, R. Polydactylism in cats. Vet. Med. Small. Anim. Clin., v.63, p.948-951, 1968.

STANEK, C.; HANTAK, E. Bilateral Atavistic Polydactyly in a Colt and its Dam. Equine Vet. J., v.18, p.76-79, 1986.

STRAUSS, G. Polydaktylie und Arthrogryposis als Ursache einer Dystokie bei einem Vikunja (Lama vicugna) (Polydactyly and arthrogryposis as causes of a difficult birth in a vicuna). Der Zoologische Garten., v.72, p.372-377, 2002.
VERMUNT, J.J.; BURBIDGE, H.M.; THOMPSON, K.G. Unusual congenital deformities of the lower limb in two calves. N. Z. Vet. J., v.48, p.192-194, 2000.

VILLAGOMEZ, D.A.; ALONSO, R.A. A distinct Mendelian autosomal recessive syndrome involving the association of anotia, palate agenesis, bifid tongue, and polydactyly in the dog. Can. Vet. J., v.39, p.642643, 1998.

WEINHART, G.; GOTZ, E.; GOTZ, H.J. Polydactyly in a foal. a case report. Tierärztl Prax., v.24, p.275277, 1996.

WEINSTEIN, S.L.; BUCKWALTER, J.A. Ortopedia de Turek: princípios e sua aplicação, 5.ed. São Paulo: Manole, 2000.

WELCH, R.D. Polydactylism. In: COLAHAN, P.T. (Ed.) et al. Equine Medicine and Surgery, 5.ed. St Louis: Mosby, 1999. p.1586.

WHITTINGTON, R.J.; UNGER, D.B.; WALSON, J.M. Multiple congenital malformations of the face, nervous system and musculo-skeletal system of pigs. Aust. Vet. J., v.63, p.48-50, 1986.

ZAPATA B.; GONZAALEZ, B.A.; MARIN, J.C. et al. Finding of polydactyly in a free-ranging guanaco (Lama guanicoe). Small Rum. Res., v.76, p.220-222, 2008. 